\title{
The divergent chromosomal ars operon of Acidithiobacillus ferrooxidans is regulated by an atypical ArsR protein
}

\author{
Bronwyn G. Butchert and Douglas E. Rawlings
}

Department of

Microbiology, University of Stellenbosch, Private Bag X1, Matieland 7602,

South Africa
Author for correspondence: Douglas E. Rawlings. Tel: +27 21808 5848. Fax: +27 218085846. e-mail:der@sun.ac.za

The chromosomal arsenic-resistance (ars) operon of Acidithiobacillus ferrooxidans is atypical in that it is divergent, with its arsCR and arsBH genes transcribed in opposite directions. Furthermore, the amino-acid sequence of the putative ArsR-like regulator of the ars operon is not conserved in regions that have been shown to be responsible for binding to arsenic. Instead, the ArsR-like protein of At. ferrooxidans is related to a group of unstudied ArsRlike proteins that have been found to be associated with chromosomal ars-like operons identified during genome-sequencing projects. Using arsB-lacZ, arsR-lacZ and arsC-lacZ fusions, it was shown that the ArsR-like protein of At. ferrooxidans is a repressor of the arsBH and arsRC genes of this organism, and that induction of gene expression took place when either AsIII (arsenite) or AsV (arsenate) were added. Deletion of 19 aa from the $C$ terminus of the 118 aa ArsR protein did not affect the regulation of its activity, but deletion of an additional 28 aa inactivated ArsR. Northern-blot hybridization suggested that on induction of expression, the arsRC genes were transcribed in greater quantities than the arsBH genes, but that the level of induction was not affected by the form of arsenic added (AsIII or AsV).

Keywords: arsenic resistance, ArsR-like proteins, gene regulation

\section{INTRODUCTION}

Acidithiobacillus ferrooxidans has been shown to contain genes for arsenic resistance on its chromosome (Butcher et al., 2000). Arsenic-resistance genes have been found on both the chromosomes and plasmids of various bacteria, and arsenic resistance has been reviewed extensively (Rosen, 1999; Xu et al., 1998; Tsai et al., 1997; Silver \& Phung, 1996; Cervantes et al., 1994). These arsenic-resistance genes are usually arranged in an operon, the two most common forms of which contain either three (arsRBC) or five (arsRDABC) genes. The ArsR and ArsD proteins are trans-acting regulators. ArsR is a repressor that controls the basal level of expression in response to the presence of arsenite, whereas ArsD controls the upper level of expression of the operon. ArsA is an ATPase that forms a complex with the ArsB transmembrane protein to pump arsenite out of the cell. Another gene, ars $H$, has been found in Yersinia enterocolitica (Neyt et al., 1997); the function

\footnotetext{
† Present address: Department of Biology, Williams College, Williamstown, Massachusetts 01267, USA.
}

of this gene is unknown, but it was shown to be required for resistance to arsenite and arsenate in Y. enterocolitica.

The arsenic-resistance (ars) operon from At. ferrooxidans was found to consist of two divergent elements, $\operatorname{ars} R C$ and $\operatorname{ars} B H$. The genes within these elements were shown to confer arsenic resistance upon an Escherichia coli ars mutant, AW3110 (Butcher et al., 2000). However, $\operatorname{ars} H$ was not required for arsenic resistance in $E$. coli and it is not yet known whether it is required in At. ferrooxidans. The unusual divergent arrangement of the ars operon of At. ferrooxidans has raised questions about the regulation of its genes. The finding that the $\operatorname{ars} B$ and $\operatorname{ars} C$ genes are located on divergent elements may mean that they are independently regulated. The putative ArsR protein from At. ferrooxidans only had weak homology to ArsR proteins from well-studied arsenic-resistance operons and did not contain the conserved metal-binding box, ELCVCDL, to which the arsenite inducer binds (Shi et al., 1994, 1996). Therefore, it was unclear whether the putative ArsR protein coded for within the At. ferrooxidans operon was able to regulate these genes in response to arsenite. 
Table 1. Bacterial strains, plasmids, primers and probes used in this study

\begin{tabular}{|c|c|c|}
\hline $\begin{array}{l}\text { Strain/plasmid/ } \\
\text { primer/probe }\end{array}$ & Description & Reference/source* \\
\hline \multicolumn{3}{|l|}{ Strain } \\
\hline E. coli W3110 & $\mathrm{K}-12 \mathrm{~F}^{-} \mathrm{IN}(r r n D-r r n E)$ & B. Rosen \\
\hline E. coli AW3110 & 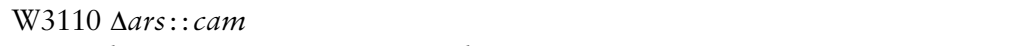 & Carlin et al. (1995) \\
\hline E. coli $\mathrm{CSH} 50 \mathrm{I}^{\mathrm{q}}$ & rspL $\Delta($ lac-pro $)\left(\mathrm{F}^{\prime}\right.$ traD36 proAB lac $\left.{ }^{\mathrm{a}} \Delta \mathrm{M} 15\right)$ & $\begin{array}{l}\text { Smith \& Rawlings } \\
\text { (1998) }\end{array}$ \\
\hline E. coli ACSH50I ${ }^{\mathrm{q}}$ & $\mathrm{CSH} 0 \mathrm{I}^{\mathrm{a}} \Delta$ ars : : cam & This work \\
\hline $\begin{array}{l}\text { At. ferrooxidans } \\
\text { ATCC } 33020\end{array}$ & Wild-type & ATCC \\
\hline \multicolumn{3}{|l|}{ Plasmid } \\
\hline pBlusecript(SK) & $\mathrm{Amp}^{\mathrm{R}} ;$ lac $Z^{\prime}$ ColE1 replicon & Stratagene \\
\hline pMC1403 & $\mathrm{Amp}^{\mathrm{R}}$; promoterless lacZYA operon ColE1 replicon & Casadaban et al. (1983) \\
\hline pKK $223-3$ & $\mathrm{Amp}^{\mathrm{R}} ; \mathrm{P}_{t a c}$ ColE1 replicon & Pharmacia Biotech \\
\hline pGL10 & $\mathrm{Kan}^{\mathrm{R}}$; RK2/RP4 replicon & A. Toukdarian \\
\hline pACYC184 & $\mathrm{Tet}^{\mathrm{R}} \mathrm{Cm}^{\mathrm{R}} ; \mathrm{p} 15 \mathrm{~A}$ replicon & Chang et al. (1978) \\
\hline pTfarsCRBH-ACYC & $\begin{array}{l}\text { Tet }^{\mathrm{R}} ; 5 \cdot 3 \mathrm{~kb} \text { HindIII-StuI fragment containing ars genes cloned into } \\
\text { pBluescript cut with EcoRV-HindIII, which was then cut with EcoRI and } \\
\text { cloned into pACYC cut with EcoRI }\end{array}$ & This study $\dagger$ \\
\hline pTfarsCRB-ACYC & $\begin{array}{l}\text { Tet }{ }^{\mathrm{R}} \text {; EcoRV-XbaI fragment of pTfars } 1 \mathrm{~b} \text {, containing the } 5^{\prime} \text { end of arsB } \\
\text { and the whole of arsH, was replaced with the EcoRV-XbaI fragment of } \\
\text { pBB08, containing only the } 5^{\prime} \text { end of ars } H \text {, which was then cloned into } \\
\text { pACYC }\end{array}$ & This study \\
\hline pTfarsBH-ACYC & $\begin{array}{l}\mathrm{Tet}^{\mathrm{R}} ; K p n \mathrm{I} \text { deletion of pTfarsCBH, cut with } K p n \mathrm{I} \text {, blunted and cut with } \\
\text { EcoRI, which was then cloned into pACYC cut with } S c a \text { I and EcoRI }\end{array}$ & This study \\
\hline pTfarsB-ACYC & $\begin{array}{l}\text { Tet }^{\mathrm{R}} \text {; Pst I deletion of pTfarsBH, which was then cut with } K p n \mathrm{I} \text {, blunted, } \\
\text { cut with BamHI and cloned into pACYC cut with } S c a \mathrm{I} \text { and BclI }\end{array}$ & This study $\dagger$ \\
\hline pTfarsC-ACYC & $\begin{array}{l}\text { Tet }^{\mathrm{R}} \text {; PCR product containing ars } C \text { cloned into pBluescript, which was } \\
\text { then cut with HindIII, blunted, cut with EcoRI and the fragment } \\
\text { containing ars } C \text { cloned into pACYC cut with } S c a \text { I and EcoRI }\end{array}$ & This study† \\
\hline pTacArsR-ACYC & $\begin{array}{l}\text { Tet }^{\mathrm{R}} \text {; PCR product from arsR-eco and arsR-hind primers cloned into } \\
\text { pKK223-3 cut with EcoRI and HindIII; } \mathrm{P}_{t a c}-\text { arsR was then excised with } \\
\text { Bam HI and } S c a \mathrm{I} \text { and cloned into pACYC cut with } B c l \mathrm{I} \text { and } S c a \mathrm{I}\end{array}$ & This study \\
\hline pTacArsH-ACYC & $\begin{array}{l}\text { Tet }^{\mathrm{R}} \text {; PCR product from ARSHF and ARSHR primers cut with EcoRI and } \\
H \text { indIII cloned into pKK223-3 cut with the same restriction enzymes; } \\
\mathrm{P}_{t a c}-a r s H \text { was then excised with } B a m \mathrm{HI} \text { and } S c a \text { I and cloned into pACYC } \\
\text { cut with } B c l \text { I and } S c a \text { I }\end{array}$ & This study \\
\hline pBlacZ & $A m p^{\mathrm{R}}$; arsB-lacZ fusion, 99 aa of ArsR (see Fig. 1 and text) & This study \\
\hline pB2lacZ & 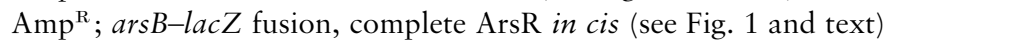 & This study \\
\hline pB2lacZ-GL10 & $\mathrm{Kan}^{\mathrm{R}}$; as above but in low-copy-number vector pGL10 & This study \\
\hline pB3lacZ & $\mathrm{Amp}^{\mathrm{R}}$; arsB-lacZ fusion, 71 aa of ArsR (see Fig. 1 and text) & This study \\
\hline pB3lacZ-GL10 & $\mathrm{Kan}^{\mathrm{R}}$; as above but in low-copy-number vector pGL10 & This study \\
\hline pRlacZ & $A m p^{R} ; \operatorname{ars} R-l a c Z$ fusion (see Fig. 1 and text) & This study \\
\hline pRlacC-GL10 & $\mathrm{Kan}^{\mathrm{R}}$; as above but in low-copy-number vector pGL10 & This study \\
\hline pClacZ & $\mathrm{Amp}^{\mathrm{R}}$; ars $C-$-lacZ fusion, ars $R$ in cis but no ars $R$ promoter & This study \\
\hline pClacZ-GL10 & $\mathrm{Kan}^{\mathrm{R}}$; as above but in low-copy-number vector pGL10 & This study \\
\hline pC2lacZ & $\mathrm{Amp}^{\mathrm{R}}$; ars $C-$-lacZ fusion, arsR in cis with ars $R$ promoter & This study \\
\hline pC2mlacZ-GL10 & $\mathrm{Kan}^{\mathrm{R}}$; as above but in low-copy-number vector pGL10 & This study \\
\hline \multicolumn{3}{|l|}{ Primers } \\
\hline BBARSB & (BamHI) 5'-GCGGATCCAGGGTGACGAGAAATATGGC-3' & Butcher et al. (2000) \\
\hline BBARSC & (BamHI) 5'-GCGGATCCGGGGTTTTCATCACTGG-3' & Butcher et al. (2000) \\
\hline RLACZE & (EcoRI) 5'-GGGAATTCCTTCGCGCTGCACGGTGA-3' & This study \\
\hline RLACZB & (BamHI) 5'-CGGGATCCATCGTATTATCCACG-3' & This study \\
\hline BLACZE & (EcoRI) 5'-CGGAATTCCCAGACAATGTGCCA-3' & This study \\
\hline arsR-hind & (HindIII) 5'-ACGAAGCTTGGGGTTTTCATCACTGG-3' & This study \\
\hline
\end{tabular}


Table 1 (continued).

\begin{tabular}{|c|c|c|}
\hline $\begin{array}{l}\text { Strain/plasmid/ } \\
\text { primer/probe }\end{array}$ & Description & Reference/source* \\
\hline arsR-eco & (EcoRI) 5'-GCGAATTCATGGAACCACTACAAGACCC-3' & This study \\
\hline ARSHF & (EcoRI) 5'-GCGAATTCTGGTGGCTGCCGCTGGCTTG-3' & Butcher et al. (2000) \\
\hline ARSHR & (HindIII) 5'-GAAAGCTTGCGTACCCCCAACCTCATGCC-3' & Butcher et al. (2000) \\
\hline \multicolumn{3}{|c|}{ Probes for Northern blots } \\
\hline ars & $1.9 \mathrm{~kb}$ Pst I fragment from pTfarsCRB & This study \\
\hline $\operatorname{ars} B$ & $0 \cdot 85 \mathrm{~kb}$ NcoI fragment from pTfarsCRBH & This study \\
\hline $\operatorname{arsR}$ & PCR product using ars-eco and ars-hind primers & This study \\
\hline $\operatorname{arsH}$ & PCR product using the ARSHF and ARSHR primers & This study \\
\hline
\end{tabular}

*B. Rosen, Wayne State University, Detroit, MI, USA; ATCC, American Type Culture Collection, Manassas, VA; A. Toukdarian, University of California, San Diego, CA, USA.

† The first cloning step was carried out by Butcher et al. (2000).

Here we describe an investigation into the regulation of the chromosomal ars operon of At. ferrooxidans and into whether the ArsR-like protein of this organism is a regulator of the ars operon in response to arsenite, arsenate and antimonite.

\section{METHODS}

Bacterial strains, plasmids, primers and media. The strains, plasmids and primers used in this study are shown in Table 1. E. coli strains were grown on Luria-Bertani (LB) medium (Sambrook et al., 1989) at $30^{\circ} \mathrm{C}$. At. ferrooxidans strains were grown in tetrathionate medium (Powles et al., 1995) at $30^{\circ} \mathrm{C}$. Ampicillin $\left(100 \mu \mathrm{g} \mathrm{ml}^{-1}\right)$, chloramphenicol $\left(20 \mu \mathrm{g} \mathrm{ml}^{-1}\right)$, tetracycline $\left(20 \mu \mathrm{g} \mathrm{ml}^{-1}\right)$ and kanamycin $\left(30 \mu \mathrm{g} \mathrm{ml}^{-1}\right)$ were added to the media when required.

DNA techniques and analyses. Plasmid preparations, restriction endonuclease digestions, gel electrophoresis and cloning techniques were carried out using standard protocols (Sambrook et al., 1989). Searches for sequences related to ArsR were performed using the gapped-BLAST program of the National Center for Biotechnology Information (NCBI) (http://www.ncbi.nlm.nih.gov/BLAST/) (Altschul et al., 1997). The PC-based DNAMAN software (version 4.1; Lynnon BioSoft) was used to create the sequence alignments and homology trees.

Construction of the arsenic-sensitive lac-negative $E$. coli strain. The generalized transduction method using phage $\mathrm{P} 1_{\mathrm{vir}}$ (Miller, 1972) was used to construct this strain. Phage $\mathrm{P} 1_{\mathrm{vir}}$ was isolated from E. coli AW3110 (Carlin et al., 1995) and used to transduce E. coli $\mathrm{CSH} 50 \mathrm{I}^{\mathrm{q}}$. The transduced cells were selected on chloramphenicol and X-Gal plates. They were then checked for sensitivity to arsenic on plates containing $0.5 \mathrm{mM}$ sodium arsenite. Sensitivity indicated that the $\Delta$ ars::cam from E. coli AW3110 had replaced the ars genes from E. coli CSH50I ${ }^{\mathrm{q}}$, resulting in strain E. coli ACSH50I ${ }^{\mathrm{q}}$.

Construction of the promoter-reporter gene (lacZ) constructs. The putative promoter regions were amplified by PCR using primer pairs BBARSB/BLACZE (arsB promoter) and RLACZB/RLACZE (arsR promoter) (Table 1). The resulting PCR products were digested with BamHI and EcoRI (the restriction sites of these enzymes were included in the primers; Table 1) and ligated to the promoterless lac $Z$ gene of pMC1403, which had been digested with the same enzymes; this resulted in $\mathrm{pB} 3 \mathrm{lac} Z$ and $\mathrm{pRlac} Z$, respectively, which were translational fusions of arsB and arsR with the lacZ gene (Fig. 1). Primers BBARSB/BBARSC were used to amplify a region containing the ars $B$ promoter and the intact arsR gene (Fig. 2). This PCR product was digested with $B a m \mathrm{HI}$ and cloned into pMC1403, which had been digested with BamHI. The product could be cloned in either direction. Cloning the insert in one direction resulted in $\mathrm{pB} 2 \mathrm{lac} Z$, in which the arsB promoter was fused to lacZ of pMC1403 and the intact $\operatorname{ars} R$ gene was transcribed in the opposite direction (Fig. 1). Cloning the insert in the other direction resulted in lac $Z$ being fused in-frame at the start of ars $C$, resulting in pC2lacZ (Fig. 1). In both constructs, the arsR promoter and the intact ars $R$ gene were also included. The above PCR product was also digested with BamHI and SmaI and cloned into pMC1403, which had been digested with the same enzymes. The resulting construct, pBlacZ, contained a translational fusion of $a r s B$ with $l a c Z$ and a partly truncated $\operatorname{ars} R$ gene in cis (Fig. 1).

The translational fusions from pB3lacZ, pRlacZ, pB2lacZ and pC2lacZ were cloned into the low-copy-number, RK2-replicon-based vector pGL10. The fusions were obtained by digesting the construct with SalI, blunting this site with T4 DNA polymerase (Roche Molecular Biochemicals) and then digesting with EcoRI. The fragment containing the fusion was then cloned into pGL10, which had been digested with SmaI and EcoRI. The constructs pB3lacZ-GL10, pRlacZ-GL10, pB2lacZ-GL10 and pC2lacZ-GL10 were constructed in this way. The ars genes were also cloned into the relatively lowcopy-number, ColE1-replicon-compatible vector pACYC184, to enable expression in trans with the promoter fusion constructs (Table 1). Correct construction of all lacZ fusions was confirmed by DNA sequencing.

$\boldsymbol{\beta}$-Galactosidase assays. Overnight cultures were diluted 1:100 into fresh medium containing the appropriate antibiotics. The fresh cultures were grown at $30^{\circ} \mathrm{C}$ until an $\mathrm{OD}_{600}$ value of between 0.3 and 0.4 was obtained. Twenty-five micromolar sodium arsenite, sodium arsenate or potassium antimonite was then added to the cultures as an inducer and the cultures were grown for a further $1 \mathrm{~h}$, after which the $\beta$-galactosidase activities of the cultures were measured using the method of Miller (1972).

Isolation of total mRNA. The yeast total RNA isolation protocol of Liang \& Pretorius (1992) was used. RNA was isolated from E. coli ACSH50I ${ }^{q}$ strains carrying pTfarsCRBH- 


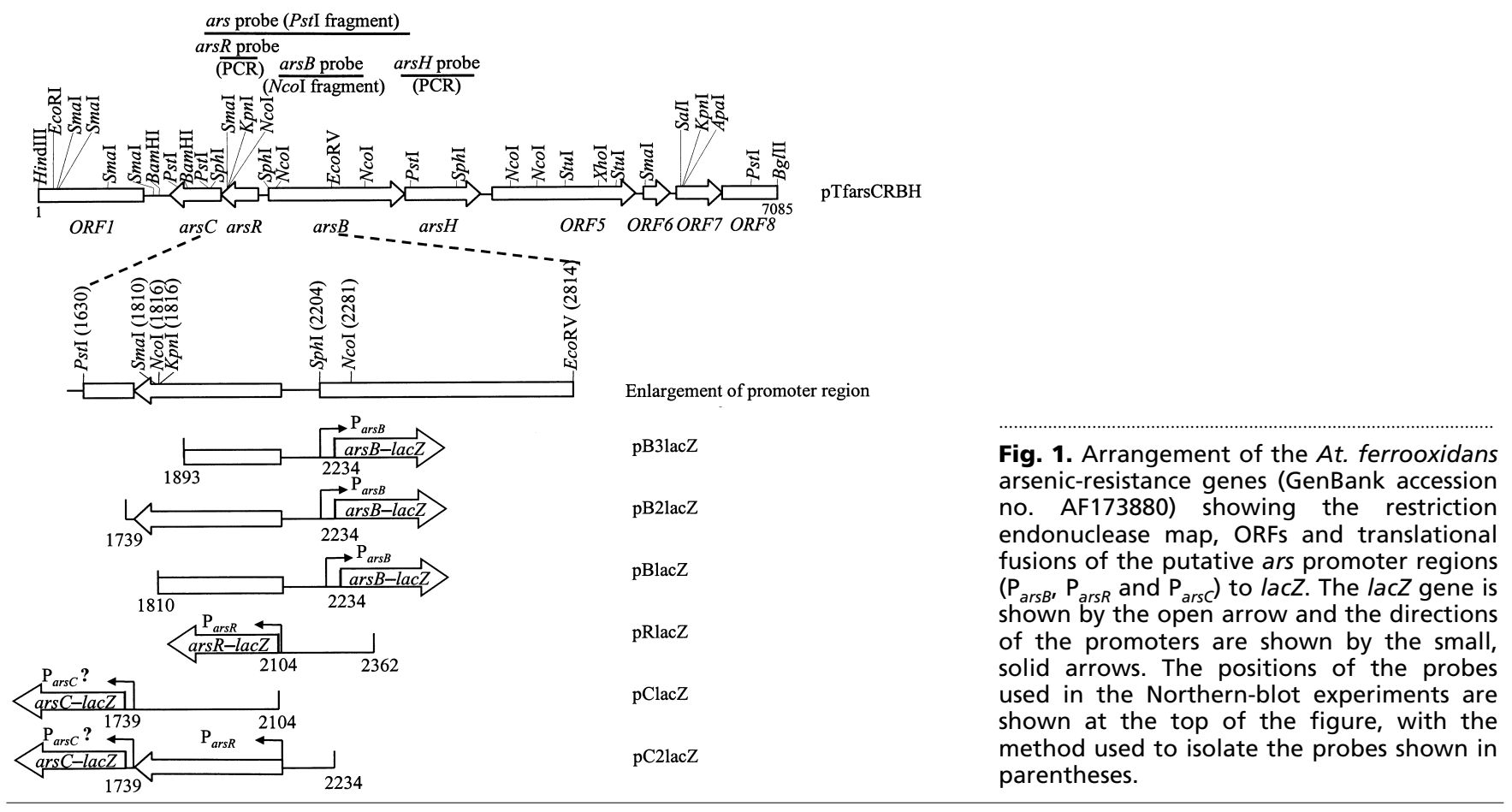

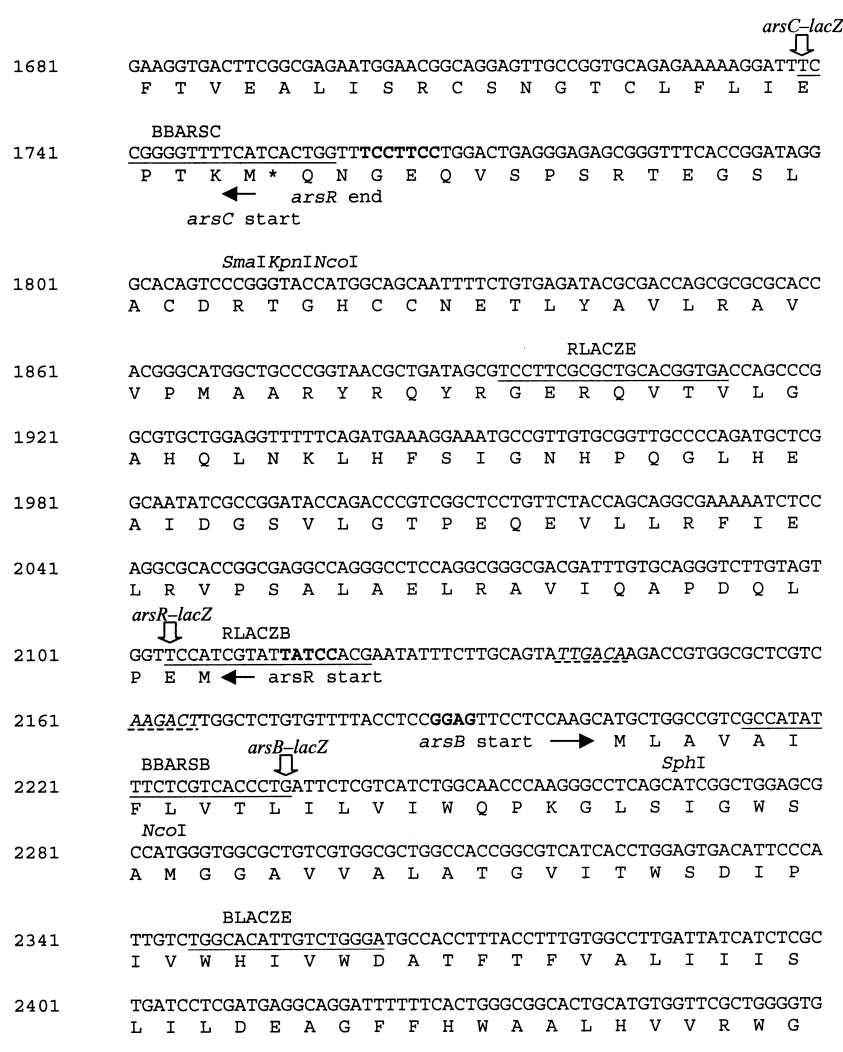

Fig. 2. Sequence of ars $R$ and the $93 \mathrm{bp}$ intergenic region, showing the locations of the primers used to create the ars-lac $Z$ fusions (underlined) and the positions of the translational fusions (open arrows). Putative ribosome-binding sites are highlighted (bold), while the broken lines underline putative -10 and -35 sequences for ars $B$ that have a good consensus for a $\sigma^{70}$ promoter. Numbering shown is according to the $A t$. ferrooxidans ars sequence (GenBank accession no. AF173880).
ACYC, which contains the complete ars operon from At. ferrooxidans. The cells were grown overnight in LB and then diluted 1:100 into fresh media containing $25 \mu \mathrm{M}$ sodium arsenite or $50 \mu \mathrm{M}$ sodium arsenate. The cells were grown at $30{ }^{\circ} \mathrm{C}$ for a further $4 \mathrm{~h}$, prior to RNA being isolated from the cultures. RNA was also isolated from At. ferrooxidans ATCC 33020. At. ferrooxidans was first grown without arsenic in $800 \mathrm{ml}$ tetrathionate medium. The cells from this culture were resuspended in $1.5 \mathrm{ml}$ acid water and used to inoculate media containing $25 \mu \mathrm{M}$ sodium arsenite, $25 \mu \mathrm{M}$ sodium arsenate or no arsenic. The resulting cultures were incubated at $30^{\circ} \mathrm{C}$ overnight and then used for RNA extractions.

Northern-blot analysis. RNA $(10 \mu \mathrm{g})$ from each sample was separated on a $1 \%$ denaturing agarose/formaldehyde gel. The gel was soaked in $20 \times$ SSC for $1 \mathrm{~h}$ and the RNA was then transferred to a Hybond-N nylon membrane under capillaryblotting in the presence of $20 \times$ SSC. The required probes (Fig. 1 and Table 1) were labelled with $\left[{ }^{32} \mathrm{P}\right] \mathrm{dATP}$ using the Random Primed DNA labelling kit (Roche Molecular Biochemicals) and hybridized to the RNA overnight at $60^{\circ} \mathrm{C}$ in hybridization buffer $(7 \%$ SDS, $1 \%$ BSA, $1 \mathrm{mM}$ EDTA, $\left.0 \cdot 25 \mathrm{M} \mathrm{Na}_{2} \mathrm{HPO}_{4}\right)$. After hydridization, the membrane was washed in $1 \times \mathrm{SSC} / 0.1 \%$ SDS and $0 \cdot 1 \times \mathrm{SSC} / 0.1 \%$ SDS; it was then exposed to X-ray film to detect the bound probe.

\section{RESULTS}

\section{Comparison of ArsR sequences}

The predicted amino-acid sequence of the putative ArsR protein from At. ferrooxidans was compared with sequences in the NCBI database using the BLAST search tool; there were no proteins with known functions that showed strong matches to the putative protein from At. ferrooxidans. ArsR from At. ferrooxidans was most similar to unstudied proteins discovered during genomesequencing projects, with the closest match (E-value of 
$3 \mathrm{e}^{-18}$ ) being to a hypothetical transcriptional regulator from Sinorhizobium meliloti (GenBank accession no. NP_385183). Putative regulators that gave the closest matches to the At. ferrooxidans ArsR protein were located adjacent to a putative membrane binding protein and often to Ars C and ArsH homologues. An alignment of the At. ferrooxidans ArsR sequence with the sequences of its nine most similar proteins (on the basis of the BLAST search results) and other known ArsR proteins was constructed (Fig. 3a). Of the nine closest matches to the ArsR protein of At. ferrooxidans from the database, none contained the conserved cysteine residues in its metal-binding domain (ELCVCDL) (Shi et al., 1996). However, there were a number of residues that were conserved throughout all of the proteins studied here, including the known ArsR proteins. These conserved residues were grouped in two regions, one before and one after the metal-binding motif previously identified in the known ArsR proteins. Besides a totally conserved arginine, serine and leucine residue within the ArsR and ArsR-like sequences, the other conserved residues were mainly hydrophobic.

Several observations could be made from the multiplesequence alignment detailed above. While the sequences of ArsR from At. ferrooxidans and its closest matches did not contain the putative metal-binding sequence, they did contain a conserved sequence $[G X(L / I) A]$ that was situated immediately downstream of the region corresponding to the experimentally established metalbinding motif. All proteins that lacked the putative metal-binding sequence had two extra residues before the second helix of the DNA-binding helix-turn-helix motif. Furthermore, all of the atypical ArsR-like proteins contained a cysteine doublet towards their Cterminals.

The sequences of the At. ferrooxidans ArsR protein and its nine closest matches clustered as a second subgroup of ArsR regulators upon homology analysis (Fig. 3b). Although all members of this subgroup shared conserved regions among themselves, and with SmtB and other known ArsR regulators, in the absence of a metalbinding domain, it was important to discover whether the atypical At. ferrooxidans ArsR protein was able to regulate the arsenic-resistance genes in response to arsenic.

\section{arsBH and arsRC promoter studies}

The arsenic-sensitive E. coli mutant strain AW3110 was wild-type for lac; hence, we constructed an E. coli strain $\left(\mathrm{ACSH} 50 \mathrm{I}^{\mathrm{q}}\right.$ ) that was both arsenic-sensitive and lacnegative. This ensured that there was no host cell background $\beta$-galactosidase activity and that there were no E. coli chromosomal ars genes present that may have interacted with the promoter-fusion constructs. E. coli ACSH50I ${ }^{\mathrm{q}}$ was used for all of the work presented here; this strain also contained $l a c l^{\mathrm{q}}$ on the $\mathrm{F}^{\prime}$ plasmid, ensuring that any genes that were added in trans and controlled by $\mathrm{P}_{t a c}$ were repressed in the absence of IPTG.
To study the expression of the ars genes we designed a number of translational gene fusions. The use of reporter genes to study gene expression should ideally be done in a homologous host, with a single gene copy integrated into the chromosome; however, this is not possible with At. ferrooxidans. To avoid many integration experiments into a heterologous $E$. coli host, all of the ars-reporter gene fusions were transferred into the lowcopy-number vector pGL10. Translational fusions were constructed where an intact arsR gene was included in cis (pB2lacZ-GL10 and pC2lacZ-GL10) and an arsBlac $Z$ fusion was constructed in which 100 aa of ArsR were included (pBlacZ) (Fig. 1). An additional arsB translational fusion was constructed, in which the promoter region with only 71 aa of ArsR was included (pB3lacZ-GL10). Other fusions are shown in Fig. 1.

\section{Expression of arsBH}

When the arsB-lac $Z$ constructs were expressed in E. coli ACSH50I ${ }^{\mathrm{q}}$ in the absence of arsenic, $\beta$-galactosidase activities of approximately 19 and 75 units, respectively, were obtained when the complete ArsR protein (pB2lacZ-GL10) and 71 aa of ArsR (pB3lacZ-GL10) were present (Fig. 4a). This indicated that in the absence of arsenic, the presence of an intact ArsR protein repressed the ars $B H$ genes by approximately fourfold. With the addition of either $25 \mu \mathrm{M}$ sodium arsenite or sodium arsenate this repression was relieved by three- to 3.5-fold provided that intact ArsR was present. When similar experiments were carried out with fusions in a higher-copy-number pMC1403-based vector, the presence of intact ArsR repressed arsB-lac $Z$ expression by about sevenfold (pB3lacZ vs pB2lacZ) in the absence of an inducer (Fig. 4b). Following the addition of $25 \mu \mathrm{M}$ sodium arsenite or sodium arsenate repression was lifted by about fivefold, while the addition of $25 \mu \mathrm{M}$ potassium antimonyl tartrate reduced repression by about threefold (Fig. 4b). An ArsR construct with 18 aa deleted from its $\mathrm{C}$ terminus (pBlacZ) gave similar levels of repression and derepression, respectively, in the absence and presence of arsenite, arsenate and antimony (Fig. $4 b)$. This indicated that the product of the ars $R$ gene was able to regulate expression from $\mathrm{P}_{\text {ars } B}$ and that while 100 aa of ArsR was sufficient for regulation of the promoter in response to arsenic and antimonite, the deletion of a further 28 aa inactivated ArsR. This additional deletion may have affected the ability of the At. ferrooxidans ArsR protein to dimerize, a feature that is essential for the functioning of the E. coli chromosomal ArsR protein and which was lost when more than 27 aa were deleted from the $\mathrm{C}$ terminus of this protein (Fig. 3) (Xu \& Rosen, 1997).

In trans expression of ars $R$ on its own under the control of $\mathrm{P}_{t a c}$ produced problems. The tac-arsR construct was toxic and host cells grew poorly at $30{ }^{\circ} \mathrm{C}$ with no growth at $37^{\circ} \mathrm{C}$. Although the addition of $2.5 \mathrm{mM} \mathrm{IPTG}$ alone reduced expression of the arsB-lacZ fusion by $20 \%$, regulation of the expression of the fusion by arsenic in the presence of IPTG was the same as in its absence. We are unsure as to the reason for this small reduction in 
(a)

SmtB

Bc chrom

R773

Bs skin

pI258

psX267

pYVe227

P. aeruginosa

NP 486806

$\mathrm{Sa}$ ZntR

Atf ArsR

NP 354498

NP_385183

NP 103579

NP_396254

NP_437556

NP 420316

NP_ 522690

NP 103576

NP_217156

SmtB

CadC

BC chrom

R773

Bs skin

pI258

pSX267

pYVe 227

$\mathrm{Pa}$ chrom

NP 486806

zntr

Atf ArsR

NP_354498

NP 385183

NP 103579

NP_396254

NP_437556

NP_420316

NP_522690

NP 103576

NP_217156

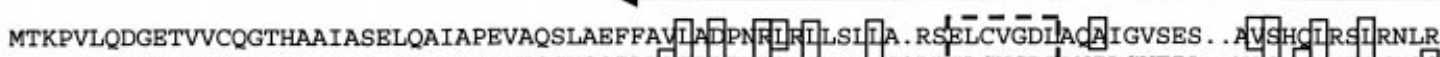
.... MKKKDTCEIFCYDEEKVNRIQGDLQTVDISGVSQILKA

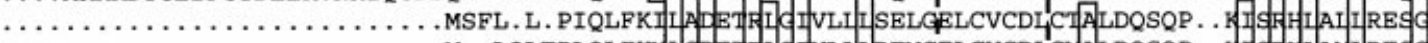

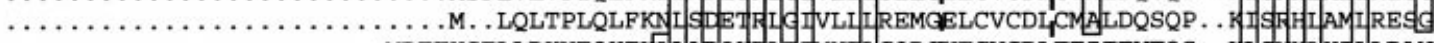

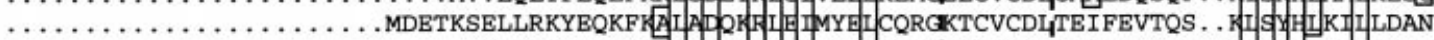

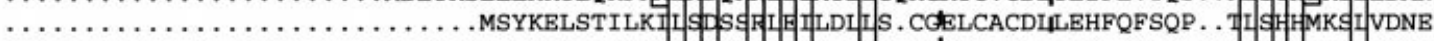

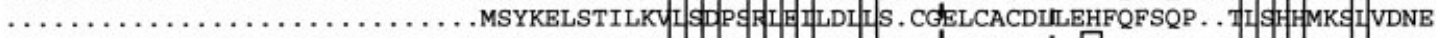

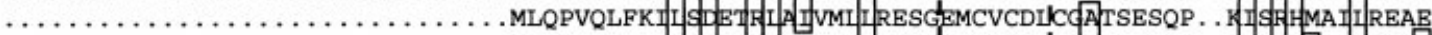

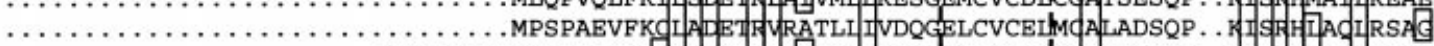

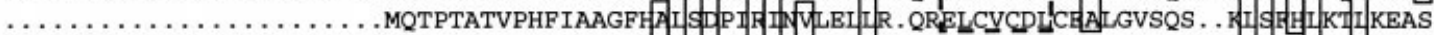
$\ldots \ldots \ldots \ldots \ldots \ldots \ldots$ MSEQYSEINTDTLERVTBI FKA I E

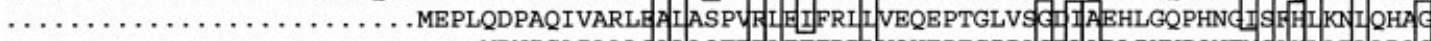

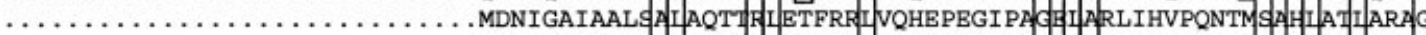

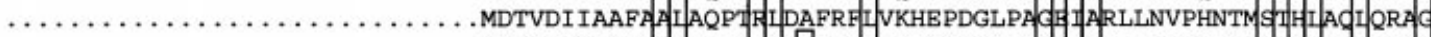

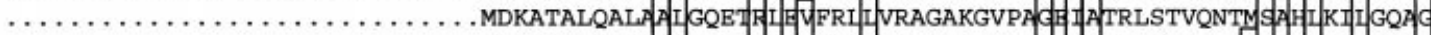

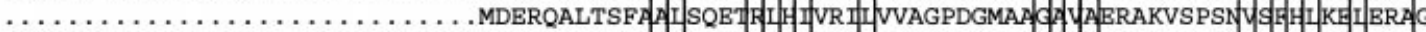

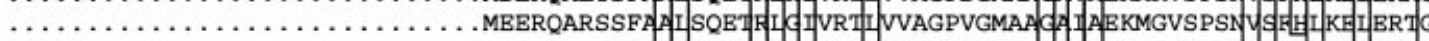

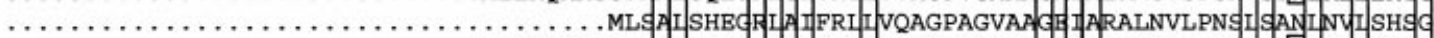

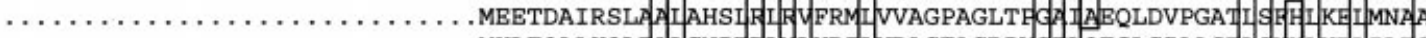
$\ldots \ldots \ldots \ldots \ldots \ldots \ldots \ldots \ldots \ldots \ldots \ldots \ldots \ldots$. . . . . . .

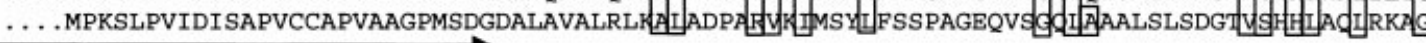

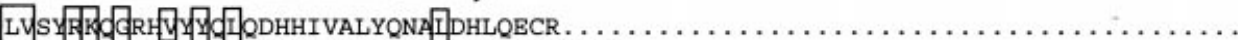

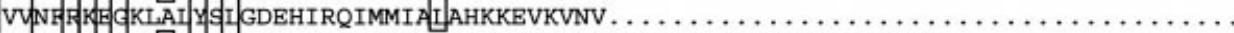

LI LD RKO KWVYH Y I I SPHI PAWAAKI IDEAWRCEQEKVQAIVRNLARQNCSGDSKNICS . . . . . . . . . II LIR LO GKW H YRI SPHI PSWAAQI IBQAWLSQQDDVQVIARKLASVNCSGSSKAVCI $\ldots \ldots \ldots \ldots \ldots$

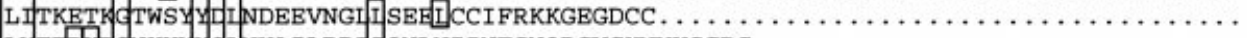

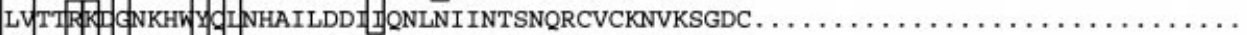
LYTIR G GNKHMYI YNHEFLDYINQNLDI INTSDQGCACKNMKSGEC $\ldots \ldots \ldots \ldots \ldots \ldots \ldots \ldots$ LVLCPRE CKWVH YRI SPHMPAWAAETITTSWHCCGKMFVSGWIN. QRHHPAEMNRTHSFNHM . . . . . .

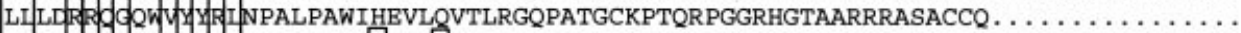

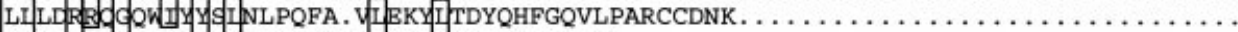

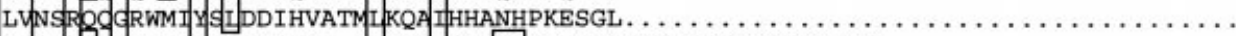

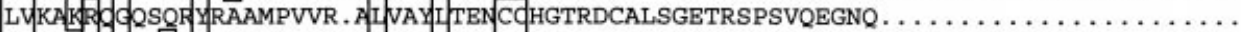
LYTVORE GRYI I YRADLEGLR . A TLF ILKIC GGATBLCAPLIABLTPCCCQEABAL . . . . . . . . . . LYKSERQSR II YRADLDGLRHYVS. F ILKCCAGHPDVCAPLVADLTPCCSPMDRPASRSHRSEGGGMDGBAADRKN

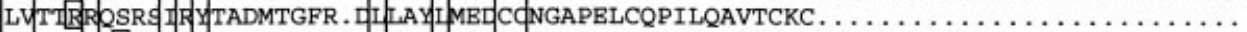

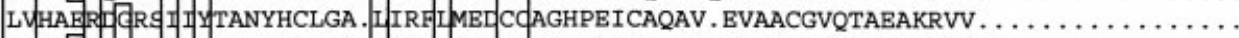

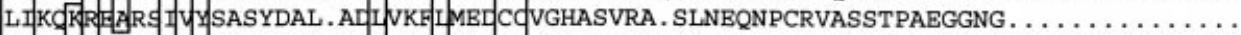

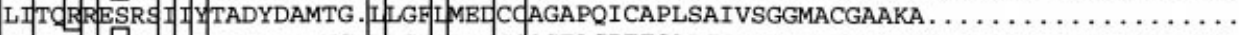

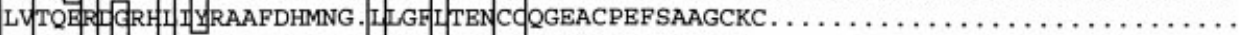

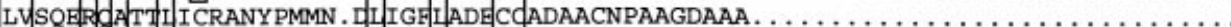

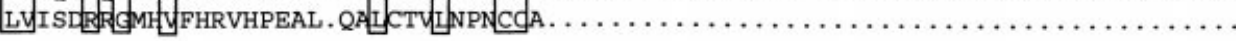

(b)

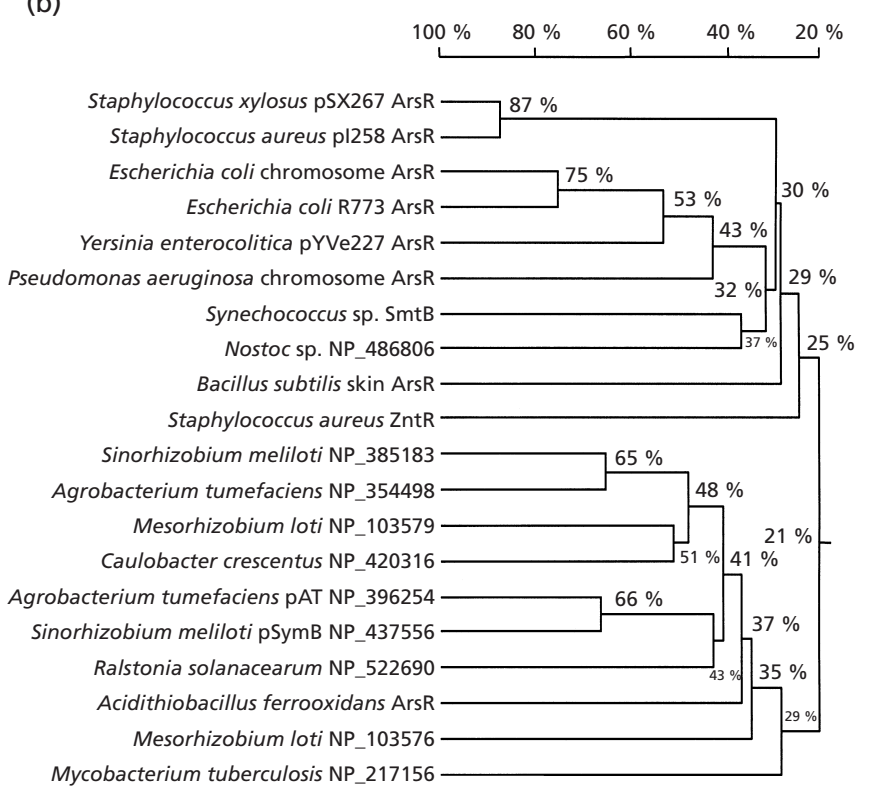

Fig. 3. For legend see facing page. 

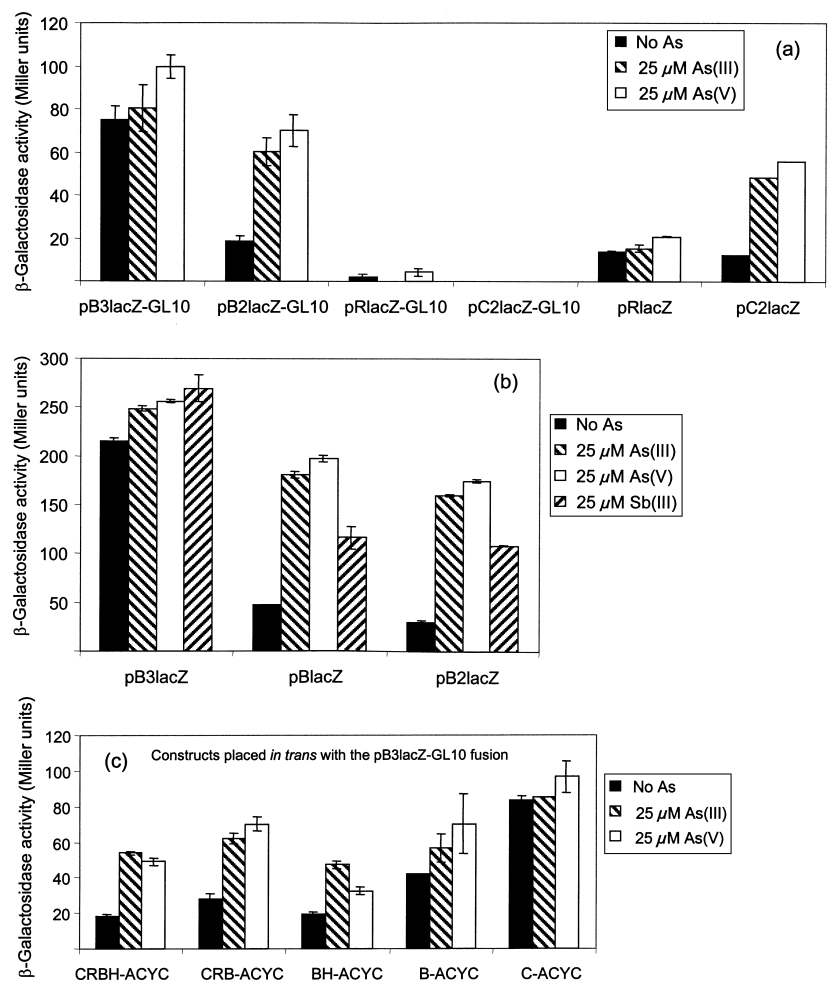

Fig. 4. (a) Expression of $\beta$-galactosidase under the control of the arsB (pB3lacZ-GL10) and arsR promoters (pRlacZ-GL10) in the presence of $25 \mu \mathrm{M}$ sodium arsenite and sodium arsenate. Also shown is the ars $B$ promoter fusion with an intact ars $R$ gene in cis (pB2lacZ-GL10) and the arsRC-lacZ fusion (pC2lacZ-GL10). These fusions were cloned into the low-copy-number vector pGL10. Fusions to arsR (pRlacZ) and arsRC (pC2lacZ) are also shown in a higher-copy-number pMC1403-based vector as expression was very low. (b) ars $B$ promoter-lac $Z$ fusions

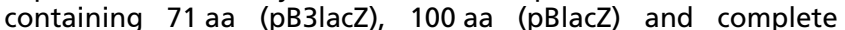
(pB2lacZ) ArsR in cis induced with $25 \mu \mathrm{M}$ sodium arsenite, sodium arsenate or antimonyl tartrate. (c) Various ars constructs added in trans with the ars $B$ promoter-lacZ fusion pB3lacZGL10. pTfarsB-ACYC and pTfarsBH-ACYC contain the same truncated yet functional ArsR as pBlacZ. All assays were carried out in triplicate and the bars represent the SD for two or more experiments.

expression caused by IPTG. However, when constructs containing arsR expressed from its own promoter, together with other ars genes (pTfarsCRBH-ACYC and
pTfarsCRB-ACYC), were added in trans with the pB3lacZ-GL10 construct, reporter gene expression was repressed to levels similar to those in pB2lacZ-GL10. This repression could be relieved by the addition of both arsenite and arsenate (Fig. 4c), although the level of expression after induction was not as high as the unrepressed $\beta$-galactosidase levels. (Note that pTfarsBH-ACYC and pTfarsB-ACYC contained the same truncated but functional arsR gene as pBlacZ).

Interestingly, when the expression of pB3lacZ-GL10 was compared with the expression of pTfarsBH-ACYC and $\mathrm{pTfarsB}-\mathrm{ACYC}$ in trans, there was a suggestion that ars $H$ may play a regulatory role in ars $B$ expression. However, attempts to investigate reporter gene expression with ars $H$ expressed on its own from a $\mathrm{P}_{\text {tac }}$ indicated that ArsH did not affect tac-arsR expression.

\section{Expression of arsRC}

When the arsR-lacZ constructs were expressed in E. coli ACSH50I ${ }^{\mathrm{q}}$ in the absence of arsenic in both a low-copynumber (pRlacZ-GL10) and a higher-copy-number vector (pRlacZ), the $\beta$-galactosidase activities were 2 and 14 Miller units, respectively (Fig. 4a). These values were more than 15 -fold less than the expression levels seen from the ars $B-l a c Z$ fusion in equivalent vectors. The levels of expression detected from $\mathrm{P}_{\text {arsR }}$ in the low-copynumber vector pRlacZ-GL10 were so low that further work was carried out only with reporter gene fusions in the higher-copy-number vector pRlacZ. As might be expected, the addition of arsenic to the ars $R-l a c Z$ fusion did not result in an increase in reporter gene activity, presumably due to the absence of a functional ArsR protein (Fig. 4a). Unexpectedly, the addition of combinations of the same ars genes, shown in Fig. 4(c), in trans did not affect the expression from pRlacZ in the presence or absence of arsenic (not shown). Therefore, it appears that if ArsR does autoregulate $\mathrm{P}_{\text {arsR }}$, either it is required in cis or there are elements downstream of the promoter that are required for induction by arsenic which were not included in pRlacZ.

An ars $C-$ lac $Z$ fusion was constructed that included ars $R$ and its putative promoter region (pC2lacZ) and a second fusion was constructed that excluded the $\mathrm{P}_{\text {ars } R}$ region (pClacZ). There was no detectable expression

Fig. 3. (a) Multiple-sequence alignment of a selection of functionally characterized ArsR proteins compared with the ArsR protein from At. ferrooxidans and its nine closest matches, as determined by the BLAST search. The arrows above the alignment indicate the region of the $E$. coli chromosomal ArsR that is required for dimerization. Also included are SmtB from Synechococcus sp. PCC7942 (GenBank accession no. S31197), ZntR from Staphylococcus aureus (AAC32484) and CadC from S. aureus pl258 (B32561). Known ArsR proteins are labelled as follows: pl258, from plasmid of Staphylococcus aureus (GenBank accession no. AAA25636); pSX267, from plasmid of Staphylococcus xylosus (AAA27587); Ec chrom, from E. coli chromosome (AAC76526); R773, from plasmid of E. coli (CAA34168); pYVe227, from plasmid of $Y$. enterocolitica (AAD16860); Bs skin, Bacillus subtilis skin element (BAA06967); P. aeruginosa, from Pseudomonas aeruginosa chromosome (AAC69642). The nine closest matches to ArsR from At. ferrooxidans are labelled with their GenBank accession numbers (see Fig. 3b). The closed boxes highlight amino acids that are conserved between at least 14 of the 21 sequences or which are found in all members of one of the two subfamilies of ArsR proteins. For the comparison, amino acids assumed to be functionally equivalent were I/L/N, R/K and G/A. The metal-binding motif found in the characterized ArsR proteins has been highlighted using broken boxes. The alignments were performed using the CLUSTAL W-based optimal alignment tool of DNAMAN. (b) Dendrogram of the proteins detailed in (a) showing the possible grouping of $A t$. ferrooxidans ArsR in a separate subclass of ArsR regulators. Values shown represent the percentage amino-acid identity between the proteins. 


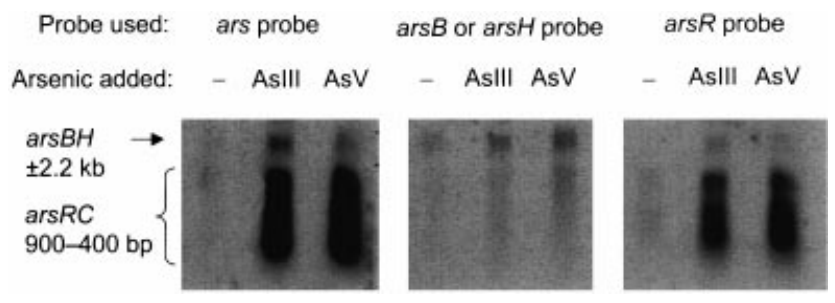

Fig. 5. Total mRNA prepared from E. coli ASCH5OI ${ }^{q}$ pTfarsCRBH$A C Y C)$ was probed with DNA probes to the arsB, arsR and arsH genes and a probe to ars $R$ and $\operatorname{ars} B$ including the intergenic region (ars probe). RNA was isolated from cells grown in the absence $(-)$ or presence of $25 \mu \mathrm{M}$ sodium arsenite (AsIII) or $50 \mu \mathrm{M}$ sodium arsenate (AsV). The positions and sizes of the transcripts are marked with arrows.

from pClacZ, which suggested that ars $C$ does not have a promoter of its own but is transcribed together with arsR (results not shown). The arsC-lacZ fusion pC2lacZ, which contained an intact ars $R$ gene, did respond to the addition of arsenic, and expression by this fusion was induced four- and $4 \cdot 5$-fold in the presence of $25 \mu \mathrm{M}$ arsenite and arsenate, respectively (Fig. 4a).

\section{Number and size of transcripts}

To investigate whether there were two mRNA transcripts for the ars operon, and to estimate their sizes and whether they were regulated differently by arsenate and arsenite in E. coli, Northern-blot analysis was performed. Total RNA was prepared from E. coli ACSH50I ${ }^{\mathrm{q}}$ (pTfarsCRBH-ACYC) cells. Probes for the $\operatorname{ars} R$, ars $B$ and $\operatorname{ars} H$ genes and a region including the ars $B$ and ars $R$ genes (ars probe) were used (Fig. 1). About $10 \mu \mathrm{g}$ of RNA was loaded into the wells of the gel shown in Fig. 5. Attempts to obtain a stronger signal by loading $30 \mu \mathrm{g}$ of RNA resulted in an increase in the signal from the degraded transcript smear and blot resolution was not improved. When RNA was isolated from At. ferrooxidans strains no transcripts were observed; hence, we believe that the ars genes are expressed at low levels in this organism.

Two different patterns of expression were seen in E. coli ACSH50I ${ }^{\mathrm{A}}$ (pTfarsCRBH-ACYC) depending on whether $\operatorname{ars} B / \operatorname{ars} H$ or $\operatorname{ars} R$ were used as probes (Fig. 5). The size of the transcript detected using either arsB or ars $H$ as probes was about $2 \cdot 2 \mathrm{~kb}$, while the ars $R C$ transcript was a smear of about $400-950 \mathrm{bp}$. When the ars probe (containing arsR and ars $B$ ) was used, two transcripts of approximately the aforementioned sizes were visible. The calculated sizes of the regions encompassing the $\operatorname{ars} B H$ and arsRC ORFs were 2038 and $847 \mathrm{bp}$, respectively, which is consistent with the sizes of the transcripts observed. It was also clear that the quantity of both transcripts, but especially that of arsRC, increased when cells were grown in the presence of either arsenite or arsenate. No clear differences in the levels of arsenite-induced expression compared to arsenate-induced expression were observed. When a probe from the tetracycline gene of pACYC184 was used for Northern blotting, a signal $(1.2 \mathrm{~kb})$ of about equal intensity was observed in all three lanes, indicating that approximately equal amounts of RNA had been loaded into each lane (results not shown). Therefore, the approximately equal levels of induction observed irrespective of whether arsenite or arsenate was used are in agreement with the results from the reporter gene fusion experiments.

\section{DISCUSSION}

The arsenic-resistance genes from At. ferrooxidans have an unusual divergent arrangement, with the two major genes, $\operatorname{ars} B$ (encoding the arsenite membrane pump) and ars $C$ (encoding the arsenate reductase), transcribed in opposite directions. A gene showing similarity to the regulatory gene ars $R$ was found in the middle of these genes, which was transcribed in the same direction as ars $C$. While members of the family of ArsR regulatory proteins often show low similarity to each other (e.g. the ArsR proteins from staphylococcal plasmids are only approximately $30 \%$ identical to those from the E. coli chromosome and plasmid R773), those that have been functionally investigated all contain a highly conserved metal-binding domain with an ELCVCDLC consensus sequence. Shi et al. (1994, 1996) found that the three cysteine residues of this consensus sequence were able to interact with arsenite. Mutations in the first two cysteine residues resulted in a protein that was able to bind the promoter and repress expression, but it was not able to undergo the conformational change required for its release from the promoter in response to an inducer. The finding that the putative At. ferrooxidans ArsR protein and its closest matches are located adjacent to a putative membrane protein (and often to ArsC and ArsH homologues) provides circumstantial evidence that these proteins are ars regulators. However, none of the putative ArsR proteins contained the conserved metal-binding motif, with the only cysteine residues being at the $\mathrm{C}$ termini of these proteins. Nevertheless, we found that mRNA production was increased in response to the presence of arsenite and arsenate. This indicated that regulation of the ars genes occurred at the transcriptional level and we showed, using promoter fusions to a $\beta$-galactosidase reporter gene (lacZ), that this regulation was controlled by the atypical ArsR protein of At.ferrooxidans. This suggests that the other related, but unstudied, ArsR-like proteins discovered during genome-sequencing projects may do the same. However, the inducer-metalloid binding mechanism of the two families of ArsR regulator proteins may be different. For example, the ars operon of E. coli plasmid R773 was derepressed by arsenite and antimony but not by arsenate (Wu \& Rosen, 1993). It is thought that induction by arsenate takes place after its reduction to arsenite, typically by the ArsC reductase. The At. ferrooxidans ArsR protein appeared to respond to arsenate in the E. coli ars deletion mutant containing no ArsC. This suggests that either the ability of arsenate to bind the At. ferrooxidans ArsR is different, or some 
other mechanism exists for the reduction of sufficient arsenate to arsenite, which then serves as the inducer. In a study on ZntR from the chromosome of $S$. aureus (Singh et al., 1999), it was reported that this protein was able to bind to the promoter of the zinc-resistance operon and that this binding was inhibited in the presence of zinc, even though ZntR did not contain any cysteine residues (Fig. 3a) - this protein is related to the ArsR family of regulatory proteins (Fig. 3b).

The ArsR regulators are part of the ArsR family of metalloregulatory proteins, which also includes CadC (the regulator of the $\mathrm{Cd}^{2+}$-efflux ATPase) and $\mathrm{SmtB}$ (the regulator of the metallothionein $\mathrm{SmtA}$ protein in response to the presence of zinc). In this study, we found that although ArsR from At. ferrooxidans does not contain the conserved metal-binding domain, there are many other strongly conserved areas within its sequence and the sequences of $\mathrm{SmtB}$, other known ArsR proteins and its closest matches from the BLAST search. The crystal structure of $\mathrm{SmtB}$ has been determined and has been shown to have a winged helix-turn-helix (HTH) structure (Cook et al., 1998). The secondary structure of $\mathrm{SmtB}$ consists of four $\alpha$-helices and two $\beta$-sheets, with the $\beta$-sheets having been shown to form a hairpin structure. The conserved Gly-X-X motif (where X represents a large hydrophobic residue) at the end of the DNA-binding HTH domain is found in many of the proteins shown in Fig. 3(a). Cook et al. (1998) believe that these hydrophobic residues are important for anchoring the $\beta$-sheet that follows. Secondary-structure predictions indicate that many of these general features appear to be present in the second family of ArsR-like regulators. However, the absence of the established metal-binding motif and the observation that there are two extra amino-acid residues before the conserved hydrophobic region corresponding to the second helix of the HTH domain argue against a detailed extrapolation from the secondary structure of SmtB to the ArsR protein of At. ferrooxidans.

We have shown that at least two RNA transcripts, one corresponding to ars $\mathrm{BH}$ and the other corresponding to $\operatorname{ars} R C$, were induced in the presence of arsenite and arsenate. Both Northern hybridization and reporter gene studies indicated that the level of expression from $\mathrm{P}_{\text {ars } B}$ was low. Although expression was increased following induction, the levels of expression remained low. While this could be due to inefficient expression in E. coli, these low levels of expression were also observed when fusions to the ars genes from the Bacillus subtilis skin element were constructed and integrated into the chromosome (Sato \& Kobayashi, 1998). The reason for such low levels of expression could be due to the toxic nature of the ars genes. Overexpression of the E. coli ArsB protein from a $\mathrm{P}_{t a c}$ has been reported to be toxic to cells (e.g. Cai \& DuBow, 1996). Despite the low levels of expression, induction of expression by arsenite, arsenate and antimonite was observed.

The relative levels of expression from the ars $R C$ promoter gave apparently conflicting results, depending on whether Northern hybridization or reporter gene studies were used. Although the quantity of arsRC transcript seen on the Northern blot was higher than for $\operatorname{ars} B H$, the level of arsRC-lac $Z$ expression was much lower than for the arsB-lacZ fusion. The observation that the arsRC mRNA transcript was a smear may indicate that this mRNA has a higher turnover rate than arsBH mRNA. If much of the degradation takes place from the $5^{\prime}$ end, this may be the reason for the lower level of reporter gene expression. Because reporter gene expression from the arsRC promoter was so low (less than 10 Miller units), we worked with a higher-copynumber vector and obtained results that were consistent with those observed in the low-copy-number vector. Expression from an ars $R C$ promoter fused at the start of $\operatorname{ars} R$ was unresponsive to the presence of arsenic, presumably due to the absence of arsR. The addition of an arsR gene in trans did not induce expression, possibly because sequences downstream of the point of fusion were required or because the At. ferrooxidans arsR gene is not autoregulated. This inability to demonstrate autoregulation may indicate a difference between the two families of ArsR proteins and needs to be investigated further. However, when a translational fusion to $\operatorname{ars} C$ (which included ars $R$ and the ars $R C$ promoter) was investigated, expression was induced in the presence of both arsenite and arsenate. This indicated that ars $R$ was required for regulation of this promoter. As an ars $C-$-lac $Z$ fusion containing the region upstream of ars $C$ but not including the ars $R$ promoter (pClacZ) showed no $\beta$-galactosidase activity, it appears that ars $R$ and $\operatorname{ars} C$ are expressed as a single transcript. Therefore, although the two ars transcripts of the At.ferrooxidans ars operon were transcribed at different levels, both were regulated by arsenite and arsenate.

We found that the last 19 aa of the $\mathrm{C}$ terminus of At. ferrooxidans ArsR were not required for either repression or induction of the ars $\mathrm{BH}$ promoter by arsenite, arsenate or antimony. However, when a further 28 aa of ArsR in cis with the promoter fusion were deleted (leaving only $71 \mathrm{aa}$ ), expression from the promoter was constitutive. Similarly, it was shown that E. coli chromosomal ArsR- $\beta$-lactamase chimeras were constitutive when fused to regions upstream of the 79 th amino acid, but were inducible when $\beta$-lactamase was fused downstream of the 92nd amino acid (Xu et al., 1996). These positions align with amino acids 85 and 98 of the At. ferrooxidans ArsR. An interesting observation is that the double cysteines conserved in At. ferrooxidans ArsR and its closest relatives were retained in the functional truncated ArsR. Further experiments are required to show whether these residues are involved in regulation.

We were unable to find a clear role for ArsH in the regulation of the At. ferrooxidans ars genes. While the addition of constructs containing ars $\mathrm{H}$ in trans with the ars $B$ promoter fusions appeared to result in increased repression of the promoter and lower levels of induction, especially when arsenate was the inducer, these differences were small (Fig. 4b). We were unable to add the 
tac-regulated ars $H$ gene back in trans, as we found that IPTG on its own decreased expression from the At. ferrooxidans ars $B$ promoter. It is possible that the role that ArsH plays is only important in At. ferrooxidans and not in E. coli.

This is the first functional study of an ArsR regulator that is able to respond to arsenic, but which does not contain the conserved metal-binding motif. We propose that the ArsR protein from At. ferrooxidans is the first reported member of a subclass of ArsR regulators that may have a different method of binding the inducer to other ArsR regulators.

\section{ACKNOWLEDGEMENTS}

We thank Barry Rosen for the gift of E. coli strains W3110 and AW3110, Aresa Toukdarien for plasmid pGL10 and Michael S. DuBow (Institut de Génétique et Microbiologie, Bâtiment 409, Université Paris Sud, 91405 Orsay, France) for the gift of phage $\mathrm{P} 1_{\mathrm{vir}}$. This work was supported by grants from the National Research Foundation, the Technology and Human Resources for Industry Programme (Pretoria, South Africa) and BHP Billiton Minerals Technology (Randburg, South Africa).

\section{REFERENCES}

Altschul, S. F., Madden, T. L., Schaffer, A. A., Zhang, J., Zhang, Z., Miller, W. \& Lipman, D. J. (1997). Gapped BLAST and PSI-BLAST : a new generation of protein database search programs. Nucleic Acids Res 25, 3389-3402.

Butcher, B. G., Deane, S. M. \& Rawlings, D. E. (2000). The chromosomal arsenic resistance genes of Thiobacillus ferrooxidans have an unusual arrangement and confer increased arsenic and antimony resistance to Escherichia coli. Appl Environ Microbiol 66, 1826-1833.

Cai, J. \& DuBow, M. S. (1996). Expression of the Escherichia coli chromosomal ars operon. Can J Microbiol 42, 662-671.

Carlin, A., Shi, W., Dey, S. \& Rosen, B. P. (1995). The ars operon of Escherichia coli confers arsenical and antimonial resistance. J Bacteriol 177, 981-986.

Casadaban, M. J., Martinez-Arias, A., Shapira, S. K. \& Chou, J. (1983). $\beta$-Galactosidase gene fusions for analysing gene expression in Escherichia coli and yeast. Methods Enzymol 100, 293-308.

Cervantes, C., Ji, G., Ramírez, J. L. \& Silver, S. (1994). Resistance to arsenic compounds in microorganisms. FEMS Microbiol Rev 15, 355-367.

Chang, A. C. Y. \& Cohen, S. N. (1978). Construction and characterization of amplifiable multicopy DNA cloning vehicles derived from the p15A cryptic miniplasmid. J Bacteriol 134, 1141-1156.

Cook, W. J., Kar, S. R., Taylor, K. B. \& Hall, L. M. (1998). Crystal structure of the cyanobacterial metallothionein repressor SmtB : a model for metalloregulatory proteins. J Mol Biol 275, 337-346.
Liang, E. \& Pretorius, I. S. (1992). Synthesis and secretion of an Erwinia chrysanthemi pectate lyase in Saccharomyces cerevisiae regulated by different combinations of bacterial and yeast promoter and signal sequences. Gene 121, 35-45.

Miller, J. H. (1972). Experiments in Molecular Genetics. Cold Spring Harbor, NY: Cold Spring Harbor Laboratory.

Neyt, C., Iriarte, M., Thi, V. H. \& Cornelis, G. R. (1997). Virulence and arsenic resistance in Yersiniae. J Bacteriol 179, 612-619.

Powles, R. E., Deane, S. M. \& Rawlings, D. E. (1995). Molecular genetic analysis of a thioredoxin gene from Thiobacillus ferrooxidans. Microbiology 141, 2175-2181.

Rosen, B.P. (1999). Families of arsenic transporters. Trends Microbiol 7, 207-212.

Sambrook, J., Fritsch, E. F. \& Maniatis, T. (1989). Molecular Cloning: a Laboratory Manual, 2nd edn. Cold Spring Harbor, NY : Cold Spring Harbor Laboratory.

Sato, T. \& Kobayashi, Y. (1998). The ars operon in the skin element of Bacillus subtilis confers resistance to arsenate and arsenite. J Bacteriol 180, 1655-1661.

Shi, W., Wu, J. \& Rosen, B. P. (1994). Identification of a putative metal binding site in a new family of metalloregulatory proteins. J Biol Chem 269, 19826-19829.

Shi, W., Dong, J., Scott, R. A., Ksenzenko, M. Y. \& Rosen, B. P. (1996). The role of arsenic-thiol interactions in metalloregulation of the ars operon. J Biol Chem 271, 9291-9297.

Silver, S. \& Phung, L. T. (1996). Bacterial heavy metal resistance: new surprises. Annu Rev Microbiol 50, 753-789.

Singh, V. K., Xiong, A., Usgaard, T. R., Chakrabarti, S., Deora, R., Misra, T. K. \& Jayaswal, R. K. (1999). ZntR is an autoregulatory protein and negatively regulates the chromosomal zinc resistance operon znt of Staphylococcus aureus. Mol Microbiol 33, 200-207.

Smith, A. S. G. \& Rawlings, D. E. (1998). Efficiency of the pTF-Fc2 pas poison-antidote stability system in Escherichia coli is affected by the host strain, and antidote degradation requires the Lon protease. J Bacteriol 180, 5458-5462.

Tsai, K.-J., Hsu, C.-M. \& Rosen, B. P. (1997). Efflux mechanisms of resistance to cadmium, arsenic and antimony in prokaryotes and eukaryotes. Zool Stud 36, 1-16.

Wu, J. \& Rosen, B. P. (1993). Metalloregulated expression of the ars operon. J Biol Chem 268, 52-58.

Xu, C. \& Rosen, B. P. (1997). Dimerization is essential for DNA binding and repression by the ArsR metalloregulatory protein of Escherichia coli. J Biol Chem 272, 15734-15738.

Xu, C., Shi, W. \& Rosen, B. P. (1996). The chromosomal arsR gene of Escherichia coli encodes a trans-acting metalloregulatory protein. J Biol Chem 271, 2427-2432.

Xu, C., Zhou, T., Kuroda, M. \& Rosen, B. P. (1998). Metalloid resistance mechanisms in prokaryotes. J Biochem 123, 16-23.

Received 10 April 2002; revised 15 July 2002; accepted 19 August 2002. 